\title{
LHC diphoton and Z+photon Higgs signals in the Higgs triplet model with $Y=0$
}

\section{Lei Wang and Xiao-Fang Han}

Department of Physics, Yantai University, Yantai 264005, China

E-mail: leiwang@ytu.edu.cn, xfhan@mail.itp.ac.cn

AbStraCt: We study the implications of the LHC diphoton and $\mathrm{Z}+$ photon Higgs signals on the Higgs triplet model with $\mathrm{Y}=0$, which predicts two neutral CP-even Higgs bosons $h$, $H$ and a pair of charged Higgs $H^{ \pm}$. We discuss three different scenarios: (i) the observed boson is the light Higgs boson $h$; (ii) it is the heavy Higgs boson $H$; (iii) the observed signal is from the almost degenerate $h$ and $H$. We find that the inclusive Higgs diphoton rates in the first two scenarios can be enhanced or suppressed compared to the SM value, which can respectively fit the ATLAS and CMS diphoton data within $1 \sigma$ range. The inclusive $Z Z^{*}$ rates are suppressed, which are outside $1 \sigma$ range of ATLAS data and within $1 \sigma$ range of CMS data. Meanwhile, another CP-even Higgs boson production rate can be suppressed enough not to be observed at the collider. For the third scenario, the Higgs diphoton rate is suppressed, which is outside $1 \sigma$ range of ATLAS data, and the $Z Z^{*}$ rate equals to SM value approximately. In addition, we find that the two rates of $h \rightarrow \gamma \gamma$ and $h \rightarrow Z \gamma$ have the positive correlations for the three scenarios.

Keywords: Higgs Physics, Beyond Standard Model

ARXiv EPRINT: 1303.4490 


\section{Contents}

1 Introduction 1

2 Higgs triplet model with $\mathrm{Y}=0 \quad 2$

3 The Higgs diphoton and $Z \gamma$ rates at the LHC 3

3.1 Scenario I 5

$\begin{array}{llr}3.2 & \text { Scenario II } & 6\end{array}$

3.3 Scenario III 8

$\begin{array}{lll}4 \text { Conclusion } & 10\end{array}$

$\begin{array}{ll}\text { A The expressions for } \Gamma(h \rightarrow \gamma \gamma) \text { and } \Gamma(h \rightarrow Z \gamma) & 10\end{array}$

$\begin{array}{ll}\text { B The vacuum expectation values } & 11\end{array}$

\section{Introduction}

The CMS and ATLAS collaborations have announced the observation of a new boson around $125.5 \mathrm{GeV}[1,2]$, which is corroborated by the Tevatron search results [3]. The properties of this particle with large experimental uncertainties are consistent with the SM Higgs boson. Among the various signals, the diphoton and $Z Z^{*}$ are the cleanest channels of seraching for the Higgs boson. The CMS and ATLAS have presented the constraints [4, 5],

$$
\begin{array}{ll}
R_{\gamma \gamma}=0.77 \pm 0.27, & R_{Z Z^{*}}=0.92 \pm 0.28(\mathrm{CMS}), \\
R_{\gamma \gamma}=1.6 \pm 0.3, & R_{Z Z^{*}}=1.5 \pm 0.4(\mathrm{ATLAS}) .
\end{array}
$$

The CMS collaboration has released their results of the measurement of $Z \gamma$ and set an upper limit on the ratio $R_{Z \gamma}<10$ [6].

The recent Higgs data has been discussed in the SUSY models [7-27], little Higgs models [28-34] and the extensions of Higgs field models, such as the two-Higgs-doublet model [35-48], the Higgs triplet model ( $\mathrm{Y}=2$ ) [49-57], the models with septuplet [58] and color-octet scalar [59]. In this work, we will study the implications of the LHC diphoton and $Z+$ photon Higgs signals on the Higgs triplet model with $\mathrm{Y}=0$ (HTM0) [60, 61], which predicts two neutral CP-even Higgs bosons $h, H$ and a pair of charged Higgs $H^{ \pm}$. We will discuss three different scenarios: (i) the observed boson is the light Higgs $h$, and the heavy Higgs $H$ is not observed at the LHC; (ii) it is the heavy Higgs $H$, and the light Higgs $h$ is not observed at the LEP; (iii) the observed signal is from the almost degenerate $h$ and $H$. Also we will pay the particular attention to the correlations between $h \rightarrow Z_{\gamma}$ and $h \rightarrow \gamma \gamma$. Since both of the rates are loop-induced by charged particles, they should be 
closely correlated. Any new physics effects manifested in the diphoton decay should also alter the $Z \gamma$ decay [54, 62-66]

Our work is organized as follows. In section II we recapitulate the Higgs triplet model with $\mathrm{Y}=0$. In section III we discuss the LHC diphoton Higgs signal and the correlations between $h \rightarrow Z \gamma$ and $h \rightarrow \gamma \gamma$. Finally, we give our conclusion in section IV.

\section{Higgs triplet model with $\mathrm{Y}=0$}

In the HTM0, a real SU $(2)_{\mathrm{L}}$ triplet scalar field $\Sigma$ with $Y=0$ is added to the SM Lagrangian in addition to the doublet field $\Phi$. These fields can be written as

$$
\Sigma=\frac{1}{2}\left(\begin{array}{cc}
\delta^{0} & \sqrt{2} \delta^{+} \\
\sqrt{2} \delta^{-} & -\delta^{0}
\end{array}\right), \quad \Phi=\left(\begin{array}{c}
\phi^{+} \\
\phi^{0}
\end{array}\right) .
$$

The renormalizable scalar potential can be written as [67]

$$
V(\Phi, \Sigma)=-\mu^{2} \Phi^{\dagger} \Phi+\lambda_{0}\left(\Phi^{\dagger} \Phi\right)^{2}-\frac{1}{2} M_{\Sigma}^{2} F+\frac{b_{4}}{4} F^{2}+a_{1} \Phi^{\dagger} \Sigma \Phi+\frac{a_{2}}{2} \Phi^{\dagger} \Phi F(2.2
$$

where $F \equiv\left(\delta^{0}\right)^{2}+2 \delta^{+} \delta^{-}$and all the parameters are real. The Higgs doublet and triplet fields can acquire vacuum expectation values

$$
\langle\Phi\rangle=\frac{1}{\sqrt{2}}\left(\begin{array}{c}
0 \\
v_{d}
\end{array}\right), \quad\langle\Delta\rangle=\frac{1}{2}\left(\begin{array}{cc}
v_{t} & 0 \\
0 & -v_{t}
\end{array}\right)
$$

with $v^{2}=v_{d}^{2}+4 v_{t}^{2} \approx(246 \mathrm{GeV})^{2}$.

After the spontaneous symmetry breaking, the Lagrangian of eq. (2.2) predicts the four physical Higgs bosons, including two CP-even Higgs bosons $h, H$ and a pair of charged Higgs $H^{ \pm}$. These mass eigenstates are in general mixtures of the doublet and triplet fields. The mass matrixes of neutral and charged Higgs bosons are [67]

$$
\mathcal{M}_{0}^{2}=\left(\begin{array}{cc}
2 \lambda_{0} v_{d}^{2} & -a_{1} v_{d} / 2+a_{2} v_{d} v_{t} \\
-a_{1} v_{d} / 2+a_{2} v_{d} v_{t} & 2 b_{4} v_{t}^{2}+\frac{a_{1} v_{d}^{2}}{4 v_{t}}
\end{array}\right) \equiv\left(\begin{array}{cc}
A & B \\
B & C
\end{array}\right), \mathcal{M}_{ \pm}^{2}=\left(\begin{array}{cc}
a_{1} v_{t} & a_{1} v_{d} / 2 \\
a_{1} v_{d} / 2 & \frac{a_{1} v_{d}^{2}}{4 v_{t}}
\end{array}\right) .
$$

The physical mass eigenstates and the unphysical electroweak eigenstates are related by rotations through two mixing angles $\theta_{0}$ and $\theta_{+}$:

$$
\begin{aligned}
\left(\begin{array}{c}
h \\
H
\end{array}\right) & =\left(\begin{array}{cc}
\cos \theta_{0} & \sin \theta_{0} \\
-\sin \theta_{0} & \cos \theta_{0}
\end{array}\right)\left(\begin{array}{l}
\phi^{0} \\
\delta^{0}
\end{array}\right), \\
\left(\begin{array}{c}
H^{ \pm} \\
G^{ \pm}
\end{array}\right) & =\left(\begin{array}{cc}
-\sin \theta_{ \pm} & \cos \theta_{ \pm} \\
\cos \theta_{ \pm} & \sin \theta_{ \pm}
\end{array}\right)\left(\begin{array}{l}
\phi^{ \pm} \\
\delta^{ \pm}
\end{array}\right) .
\end{aligned}
$$

Where the Goldstone boson $G^{ \pm}$is eaten by the gauge bosons.

Since the experimental value of the $\rho$ parameter is near unity [68], $4 v_{t}^{2} / v_{d}^{2}$ is required to be much smaller than unity. In our calculation, $v_{t}$ is taken as $1 \mathrm{GeV}$. The mixing angle 
$\theta_{ \pm}$is proportional to $\frac{v_{t}}{v_{d}}$, therefore it is very small. The charged Higgs mass is given as

$$
M_{H^{ \pm}}^{2}=a_{1} v_{t}\left(1+\frac{v_{d}^{2}}{4 v_{t}^{2}}\right) .
$$

The neutral mixing angle $\theta_{0}$ is given as

$$
\begin{aligned}
& c_{0} \equiv \cos \theta_{0}=\frac{1}{\sqrt{2}}\left(1-\frac{A-C}{\sqrt{(A-C)^{2}+4 B^{2}}}\right)^{1 / 2}, \\
& s_{0} \equiv \sin \theta_{0}=-\frac{1}{\sqrt{2}} \frac{B}{|B|}\left(1+\frac{A-C}{\sqrt{(A-C)^{2}+4 B^{2}}}\right)^{1 / 2} .
\end{aligned}
$$

Where

$$
c_{0}>\frac{1}{\sqrt{2}} \text { for } C>A, \quad c_{0}<\frac{1}{\sqrt{2}} \text { for } \quad C<A, \quad c_{0} \rightarrow \frac{1}{\sqrt{2}} \text { for } C \rightarrow A .
$$

The neutral Higgs boson masses are given as

$$
\begin{aligned}
m_{h}^{2} & =\frac{1}{2}\left(A+C-\sqrt{(A-C)^{2}+4 B^{2}}\right), \\
m_{H}^{2} & =\frac{1}{2}\left(A+C+\sqrt{(A-C)^{2}+4 B^{2}}\right) .
\end{aligned}
$$

In our calculations, the involved Higgs couplings are listed as [67]

$$
\begin{array}{cc}
h f \bar{f}:-i \frac{m_{f}}{v_{d}} c_{0}, & H f \bar{f}: i \frac{m_{f}}{v_{d}} s_{0}, \\
Z Z h: \frac{2 i m_{Z}^{2}}{v_{d}} c_{0} g^{\mu \nu}, & Z Z H:-\frac{2 i m_{Z}^{2}}{v_{d}} s_{0} g^{\mu \nu}, \\
W^{+} W^{-} h: i g_{2}^{2}\left(\frac{1}{2} v_{d} c_{0}+2 v_{t} s_{0}\right) g^{\mu \nu}, & W^{+} W^{-} H: i g_{2}^{2}\left(-\frac{1}{2} v_{d} s_{0}+2 v_{t} c_{0}\right) g^{\mu \nu}, \\
\gamma H^{+} H^{-}: i e\left(p^{\prime}-p\right)^{\mu}, & Z H^{+} H^{-}: i\left(g_{2} c_{W}-\frac{m_{Z}}{v_{d}} s_{+}^{2}\right)\left(p^{\prime}-p\right)^{\mu}, \\
H^{+} H^{-} h:-i\left(a_{1} c_{+} s_{+} c_{0}-\frac{1}{2} a_{1} s_{+}^{2} s_{0}+a_{2} v_{d} c_{+}^{2} c_{0}+a_{2} v_{t} s_{+}^{2} s_{0}+2 b_{4} v_{t} c_{+}^{2} s_{0}+2 \lambda_{0} v_{d} s_{+}^{2} c_{0}\right), \\
H^{+} H^{-} H:-i\left(-a_{1} c_{+} s_{+} s_{0}-\frac{1}{2} a_{1} s_{+}^{2} c_{0}-a_{2} v_{d} c_{+}^{2} s_{0}+a_{2} v_{t} s_{+}^{2} c_{0}+2 b_{4} v_{t} c_{+}^{2} c_{0}-2 \lambda_{0} v_{d} s_{+}^{2} s_{0}\right) .
\end{array}
$$

Where $s_{+}=\sin \theta_{+}$and $c_{+}=\cos \theta_{+}$. All the momenta flow into the vertex.

\section{The Higgs diphoton and $Z_{\gamma}$ rates at the $\mathrm{LHC}$}

In our calculations, we take $m_{h}, m_{H}, a_{2}, b_{4}$ and $v_{d}, v_{t}$ as the input parameters, which can determine the values of $\lambda_{0}, a_{1}, m_{H^{ \pm}}$. As mentioned above, $v_{t}$ is taken as $1 \mathrm{GeV}$. The perturbativity can give the strong constraints on $a_{2}$ and $b_{4}$,

$$
-2 \sqrt{\pi} \leq a_{2} \leq 2 \sqrt{\pi}, \quad-2 \sqrt{\pi} \leq b_{4} \leq 2 \sqrt{\pi} .
$$


The electroweak $T$ parameter can give the constraints on the splitting of $m_{H}$ and $m_{H^{ \pm}}$, $\left(m_{H}-m_{H^{ \pm}}\right)^{2}<0.96 m_{W}^{2}$ [67]. Since the coupling $H^{ \pm} \bar{f}_{i} f_{j}$ is sizably suppressed by $s_{+}$, the search experiments through the top quark decay hardly give the constraints on $H^{ \pm}$. The experimental data at the LEP gives the lower bound of the charged Higgs mass, $m_{H^{ \pm}}>$ $79.3 \mathrm{GeV}[69,70]$.

We discuss three different scenarios: (I) the observed boson is the light Higgs $h, m_{h}=$ $125.5 \mathrm{GeV}$ and $135 \mathrm{GeV} \leq m_{H} \leq 500 \mathrm{GeV}$; (II) it is the heavy Higgs $H, m_{H}=125.5 \mathrm{GeV}$ and $80 \mathrm{GeV} \leq m_{h} \leq 110 \mathrm{GeV}$; (III) the observed signal is from the almost degenerate $h$ and $H, m_{h} \simeq m_{H} \simeq 125.5 \mathrm{GeV}$.

As shown in the eq. (2.11), the $h$ couplings to $f \bar{f}$ and $W W$ are proportional to $c_{0}$ while these couplings of $H$ are proportional to $s_{0}$. Due to $v_{t} \ll v_{d}$ and $s_{+} \rightarrow 0$, the $h$ couplings to $W W$ and $H^{+} H^{-}$are sensitive to $c_{0}$ while these couplings of $H$ are sensitive to $s_{0}$. Therefore, the cross sections and the decay widths of $h(H)$ normalized to SM values can be given as

$$
\begin{aligned}
\frac{\sigma(g g \rightarrow h(H))}{\sigma_{\mathrm{SM}}(g g \rightarrow h(H))} & \simeq \frac{\sigma(p p \rightarrow j j h(H))}{\sigma_{\mathrm{SM}}(p p \rightarrow j j h(H))} \\
& \simeq \frac{\sigma(p p \rightarrow V h(H))}{\sigma_{\mathrm{SM}}(p p \rightarrow V h(H))} \simeq \frac{\sigma(p p \rightarrow h(H) t \bar{t})}{\sigma_{\mathrm{SM}}(p p \rightarrow h(H) t \bar{t})} \simeq c_{0}^{2}\left(s_{0}^{2}\right), \\
\frac{\Gamma(h(H) \rightarrow f \bar{f})}{\Gamma_{\mathrm{SM}}(h(H) \rightarrow f \bar{f})} & \simeq \frac{\Gamma(h(H) \rightarrow V V)}{\Gamma_{\mathrm{SM}}(h(H) \rightarrow V V)} \simeq \frac{\Gamma(h(H) \rightarrow g g)}{\Gamma_{\mathrm{SM}}(h(H) \rightarrow g g)} \simeq c_{0}^{2}\left(s_{0}^{2}\right),
\end{aligned}
$$

where $V$ denotes $W, Z$. Compared to SM, in addition to the modified $h t \bar{t}$ and $h W W$ couplings, the charged Higgs $H^{ \pm}$will alter the decays $h \rightarrow \gamma \gamma$ and $h \rightarrow Z \gamma$ via the one-loop. The corresponding expressiones are given in the appendix A.

The Higgs boson $\gamma \gamma, Z Z^{*}$ and $Z \gamma$ rates of HTM0 normalized to the SM values are respectively defined as

$$
\begin{aligned}
R_{h(H)}(\gamma \gamma) & =\frac{\sigma(p p \rightarrow h(H))}{\sigma_{\mathrm{SM}}(p p \rightarrow h(H))} \frac{\operatorname{Br}(h(H) \rightarrow \gamma \gamma)}{B r_{\mathrm{SM}}(h(H) \rightarrow \gamma \gamma)} \\
& \simeq c_{0}^{2}\left(s_{0}^{2}\right) \frac{\Gamma(h(H) \rightarrow \gamma \gamma)}{c_{0}^{2}\left(s_{0}^{2}\right) \Gamma_{\mathrm{SM}}(h(H))} \frac{\Gamma_{\mathrm{SM}}(h(H))}{\Gamma_{\mathrm{SM}}(h(H) \rightarrow \gamma \gamma)} \simeq \frac{\Gamma(h(H) \rightarrow \gamma \gamma)}{\Gamma_{\mathrm{SM}}(h(H) \rightarrow \gamma \gamma)} \\
R_{h(H)}\left(Z Z^{*}\right) & =\frac{\sigma(p p \rightarrow h(H))}{\sigma_{\mathrm{SM}}(p p \rightarrow h(H))} \frac{B r\left(h(H) \rightarrow Z Z^{*}\right)}{B r_{\mathrm{SM}}\left(h(H) \rightarrow Z Z^{*}\right)} \\
& \simeq c_{0}^{2}\left(s_{0}^{2}\right) \frac{c_{0}^{2}\left(s_{0}^{2}\right) \Gamma_{\mathrm{SM}}\left(h(H) \rightarrow Z Z^{*}\right)}{c_{0}^{2}\left(s_{0}^{2}\right) \Gamma_{\mathrm{SM}}(h(H))} \frac{\Gamma_{\mathrm{SM}}(h(H))}{\Gamma_{\mathrm{SM}}\left(h(H) \rightarrow Z Z^{*}\right)} \simeq c_{0}^{2}\left(s_{0}^{2}\right), \\
R_{h(H)}(Z \gamma) & =\frac{\sigma(p p \rightarrow h(H))}{\sigma_{\mathrm{SM}}(p p \rightarrow h(H))} \frac{B r(h(H) \rightarrow Z \gamma)}{B r_{\mathrm{SM}}(h(H) \rightarrow Z \gamma)} \\
& \simeq c_{0}^{2}\left(s_{0}^{2}\right) \frac{\Gamma(h(H) \rightarrow Z \gamma)}{c_{0}^{2}\left(s_{0}^{2}\right) \Gamma_{\mathrm{SM}}(h(H))} \frac{\Gamma_{\mathrm{SM}}(h(H))}{\Gamma_{\mathrm{SM}}(h(H) \rightarrow Z \gamma)} \simeq \frac{\Gamma(h(H) \rightarrow Z \gamma)}{\Gamma_{\mathrm{SM}}(h(H) \rightarrow Z \gamma)}
\end{aligned}
$$

Where $\sigma(p p \rightarrow h(H))$ is the total cross section of Higgs boson. The analytic expressions in eq. (3.2) and eq. (3.3) may help us understand the Higgs production and decay well. In our numerical calculations, we take code Hdecay to consider the relevant higher order QCD and electroweak corrections [71]. 

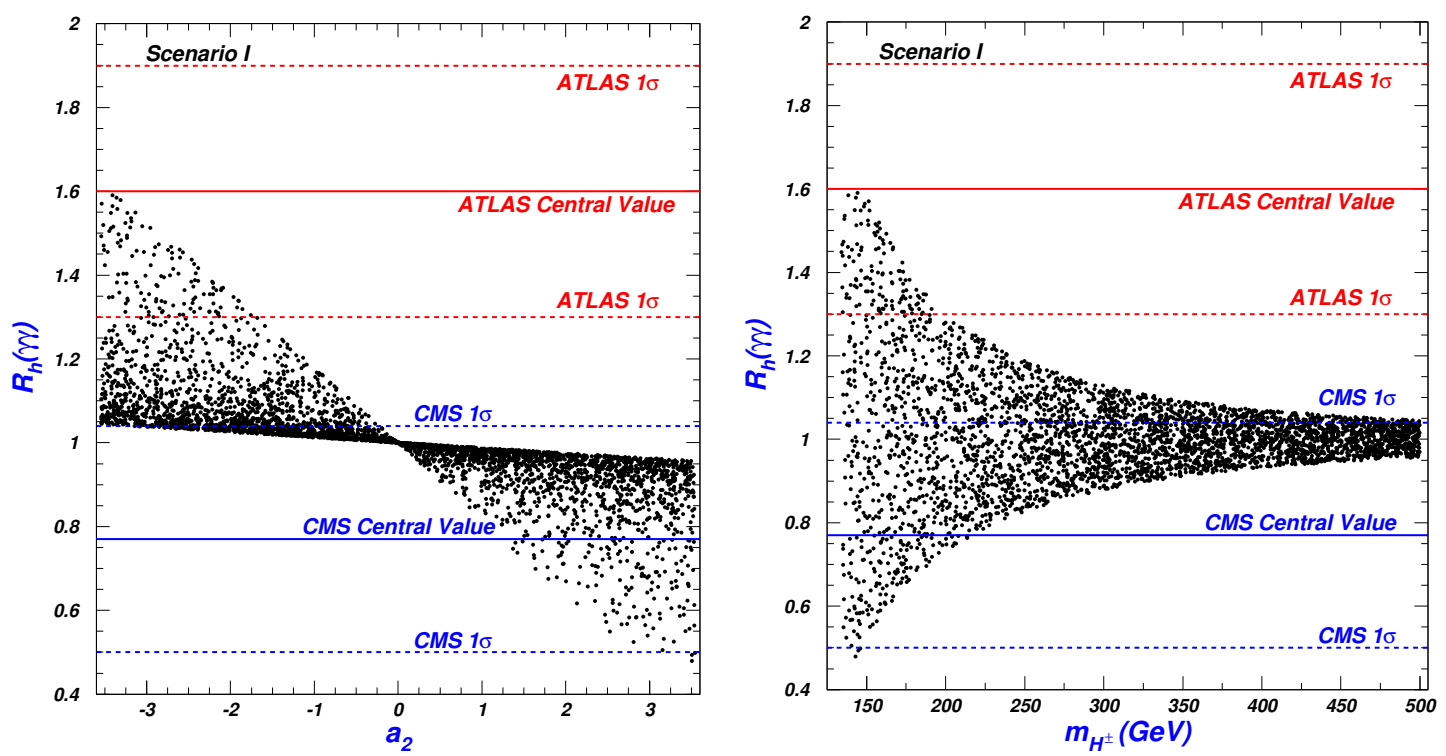

Figure 1. The scatter plots of the parameter space projected on the planes of $R_{h}(\gamma \gamma)$ versus $a_{2}$ and $R_{h}(\gamma \gamma)$ versus $m_{H^{ \pm}}$, respectively.
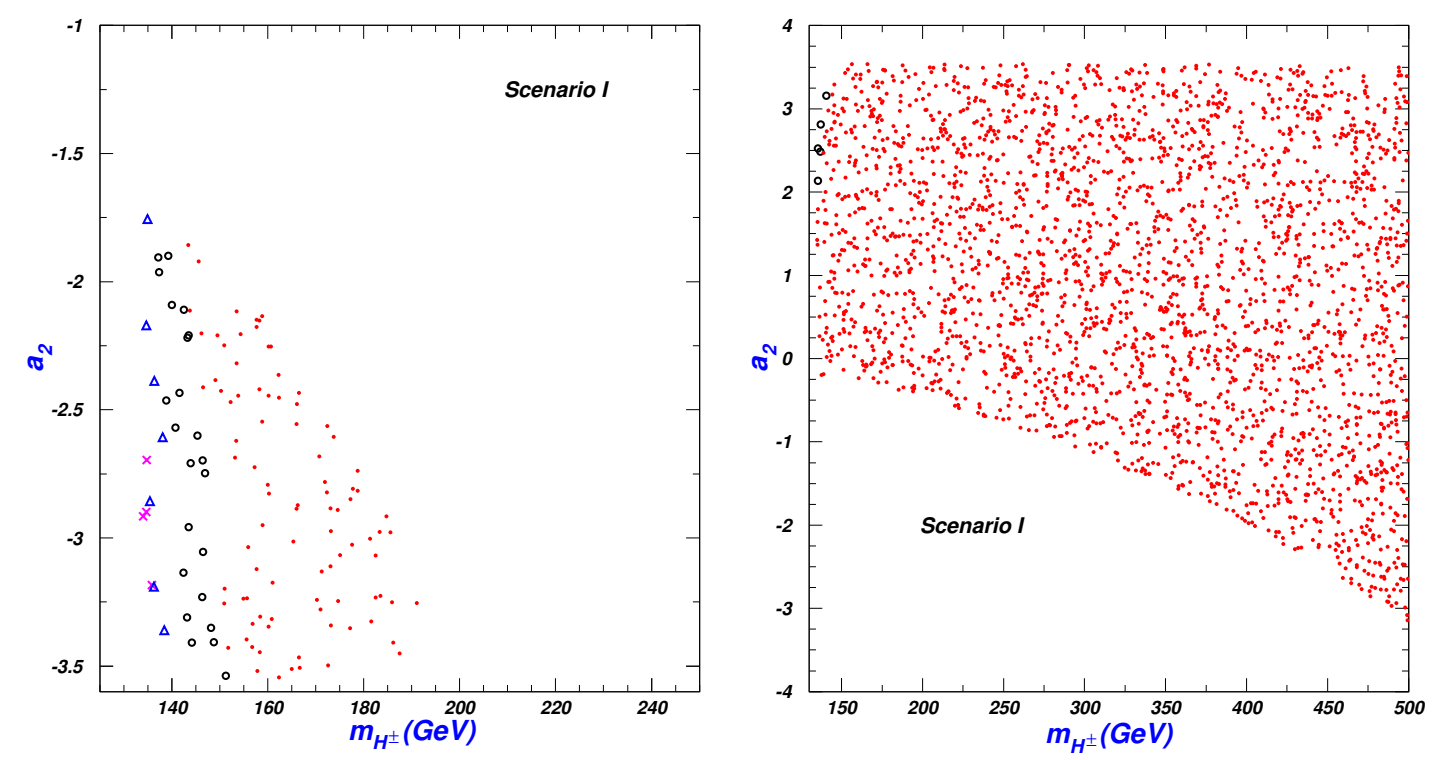

Figure 2. The scatter plots projected on the plane of $a_{2}$ versus $m_{H^{ \pm}}$. For the left panel, $R_{h}(\gamma \gamma)$ is within $1 \sigma$ range of ATLAS data. $0.86<c_{0}^{2}<0.90$ for the crosses (pink), $0.90 \leq c_{0}^{2}<0.95$ for the triangles (blue), $0.95 \leq c_{0}^{2}<0.98$ for the circles (black), and $0.98 \leq c_{0}^{2}<1.0$ for the bullets (red). The right panel is the same as the left panel, but $R_{h}(\gamma \gamma)$ is within $1 \sigma$ range of CMS data.

\subsection{Scenario I}

For the scenario I, the light Higgs $h$ is the observed boson. Since the observed $Z Z^{*}$ rate is consistent with the SM value, $c_{0}$ can not be too small. Also, it is important to make sure that the production rate of $H$ is small enough not to be detected at the LHC. Thus, to obtain a large $c_{0}$ and a small $s_{0}$, we require $C>A$ (see eq. (2.9)). 


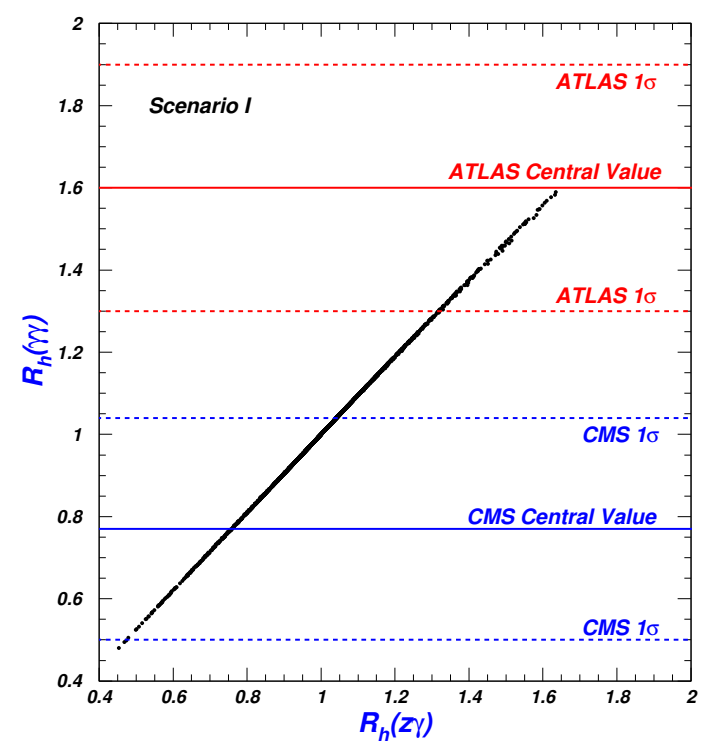

Figure 3. The scatter plots of the parameter space projected on the plane of $R_{h}(\gamma \gamma)$ versus $R_{h}(Z \gamma)$.

In figure 1 , we plot $R_{h}(\gamma \gamma)$ versus $a_{2}$ and $R_{h}(\gamma \gamma)$ versus $m_{H^{ \pm}}$, respectively. The $h$ coupling to $H^{+} H^{-}$is sensitive to the parameter $a_{2}$, which gives the additional contributions to the decay $h \rightarrow \gamma \gamma$ via one-loop. Figure 1 shows that the $H^{ \pm}$contributions to $R_{h}(\gamma \gamma)$ can interfere constructively with $W$ contributions for $a_{2}<0$ and interfere destructively for $a_{2}>0$, leading $R_{h}(\gamma \gamma)>1$ and $R_{h}(\gamma \gamma)<1$, which are respectively favored by the enhanced ATLAS diphoton data and the suppressed CMS data. The magnitude becomes sizable as the increasing of the absolute value of $a_{2}$ and the decreasing of $m_{H^{ \pm}}$.

In figure 2 , the samples with $R_{h}(\gamma \gamma)$ being within $1 \sigma$ range of ATLAS and CMS diphoton data are projected on the plane of $a_{2}$ and $m_{H^{ \pm}}$. The left panel shows that the $1 \sigma$ ATLAS diphoton data favors $-3.6<a_{2}<-1.8$ and $m_{H^{ \pm}}<190 \mathrm{GeV}$. While the CMS data favors $a_{2}>0$ and allow $a_{2}$ to be smaller than 0 for enough large $m_{H^{ \pm}}$. The left panel shows that, for $R_{h}(\gamma \gamma)$ is within $1 \sigma$ range of ATLAS diphoton data, the samples lie in the region of $c_{0}^{2}>0.86$, and the vast majority of them congregate the region of $c_{0}^{2}>0.96$. The large $m_{H^{ \pm}}$favors a large $c_{0}^{2}$. From the right panel, the value of $c_{0}^{2}$ is larger than 0.98 for $R_{h}(\gamma \gamma)$ is within $1 \sigma$ range of CMS diphoton data. Due to $R_{h}\left(Z Z^{*}\right) \simeq c_{0}^{2}$ (see eq. (3.3)), the inclusive $Z Z^{*}$ rate is outside $1 \sigma$ range of ATLAS data $(1.5 \pm 0.4)$, but within $1 \sigma$ range of CMS data $(0.92 \pm 0.28)$. Besides, for such large $c_{0}^{2}$, the corresponding $s_{0}^{2}$ is smaller than 0.14, which will suppress the production rates of $H$ at the LHC sizably (see eqs. (3.2) and (3.3)), leading that $H$ is not detected at the LHC.

Figure 3 shows $R_{h}(\gamma \gamma)$ versus $R_{h}(Z \gamma)$. We find that the two rates are positively correlated, and the behavior of $R_{h}(Z \gamma)$ is similar to that of $R_{h}(\gamma \gamma)$. Further, the prediction of $R_{h}(Z \gamma)$ equals to that of $R_{h}(\gamma \gamma)$ approximately.

\subsection{Scenario II}

For the scenario II, the heavy Higgs $H$ is the observed boson. The parameter $s_{0}$ can not be very small to make the observed $Z Z^{*}$ rate to be consistent with the experimental data. 

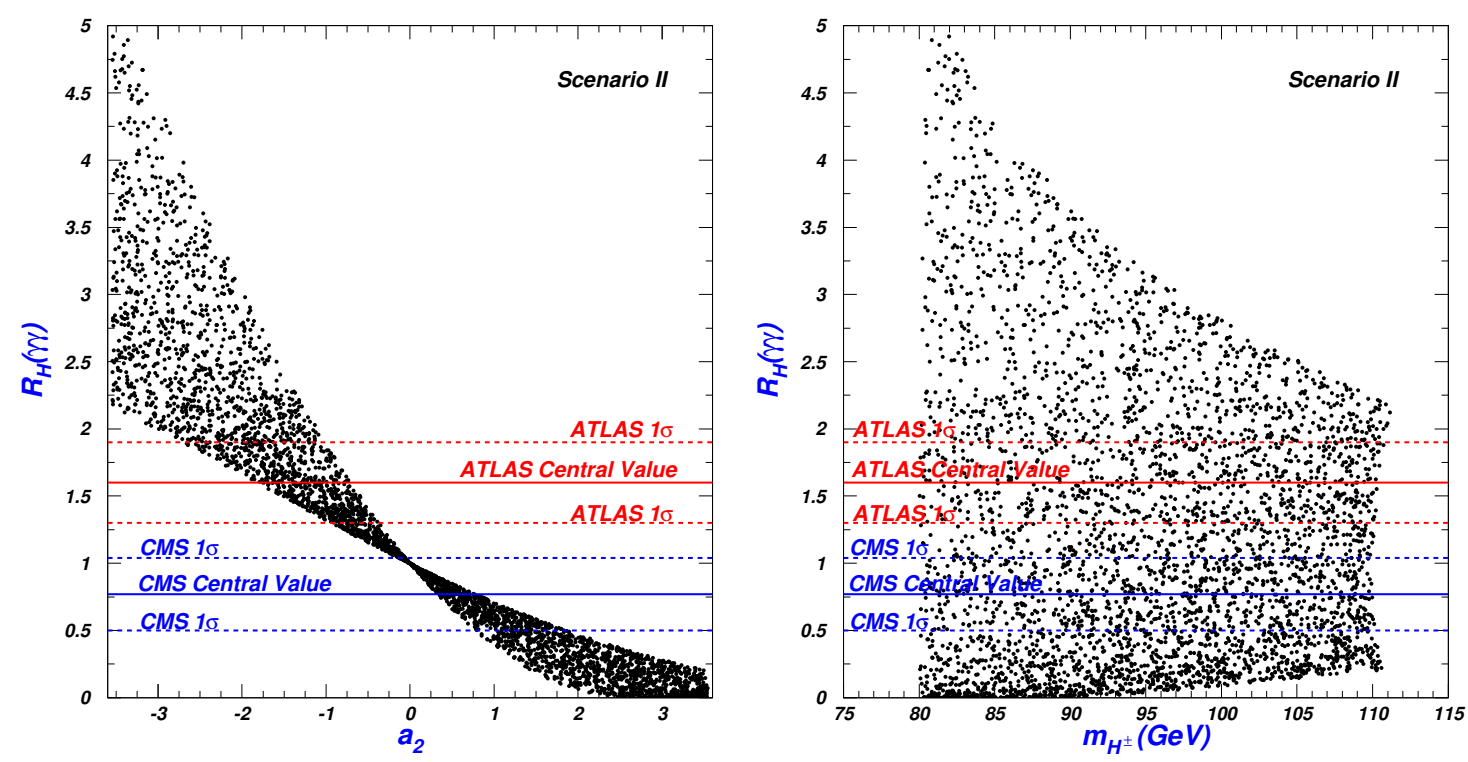

Figure 4. Same as figure 1, but for $R_{H}(\gamma \gamma)$.
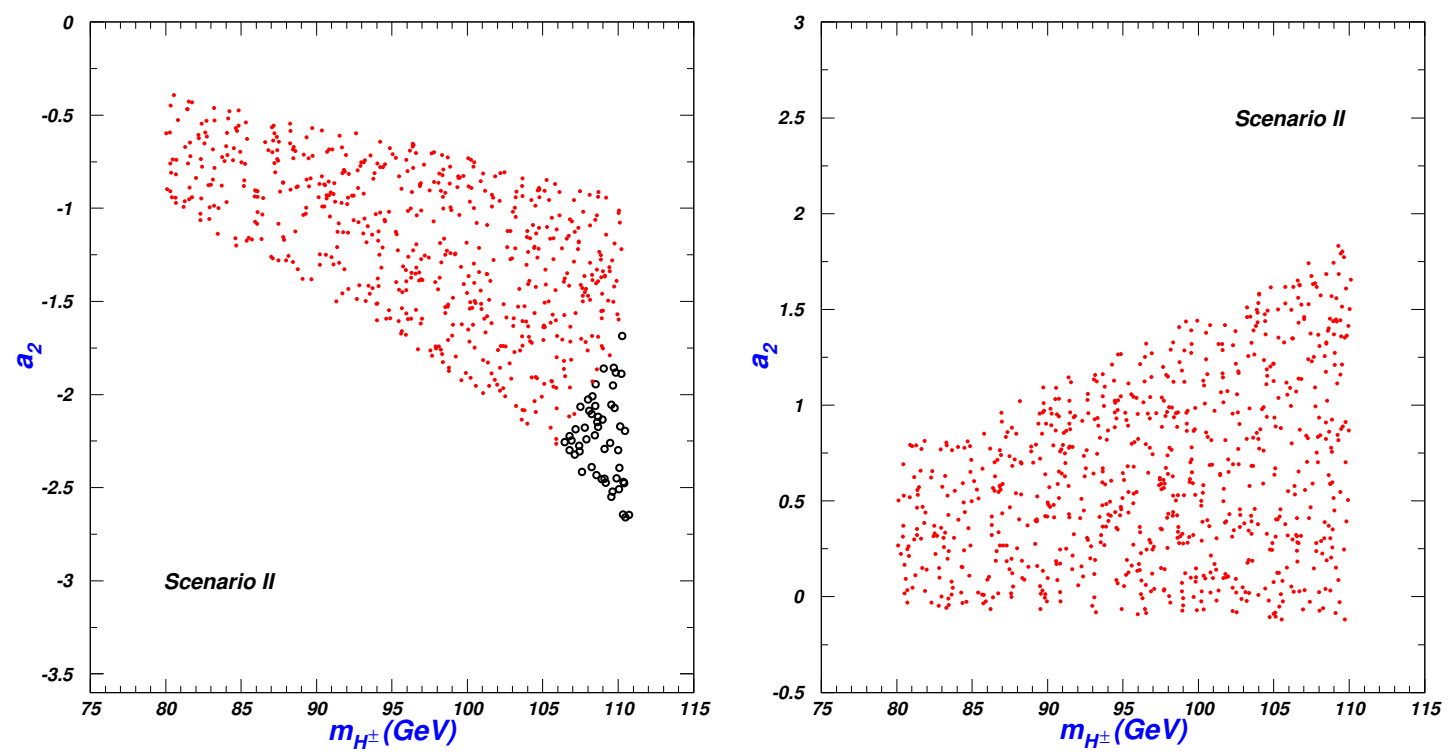

Figure 5. The Scatter plots projected on the plane of $a_{2}$ versus $m_{H^{ \pm}}$. For the left panel, $R_{H}(\gamma \gamma)$ is within $1 \sigma$ range of ATLAS data. $0.95 \leq s_{0}^{2}<0.98$ for the circles (black), and $0.98 \leq s_{0}^{2}<1.0$ for the bullets (red). The right panel is the same as the left panel, but $R_{H}(\gamma \gamma)$ is within $1 \sigma$ range of CMS data.

Besides, it is important to make sure that the production rate of $h$ is small enough not to be detected at the LEP. Thus, we require $C<A$ to obtain a large $s_{0}$ and a small $c_{0}$, (see eq. (2.9)).

In figure 4, we plot $R_{H}(\gamma \gamma)$ versus $a_{2}$ and $R_{H}(\gamma \gamma)$ versus $m_{H^{ \pm}}$, respectively. Similar to $R_{h}(\gamma \gamma), R_{H}(\gamma \gamma)$ is also larger than 1.0 for $a_{2}<0$ and smaller than 1.0 for $a_{2}>0 . R_{H}(\gamma \gamma)$ can reach 5.0 for $a_{2} \sim-3.5$ and $m_{H^{ \pm}} \sim 80 \mathrm{GeV}$, which is much larger than $R_{h}(\gamma \gamma)$ since $m_{H^{ \pm}}$for the former is smaller than that for the latter. 


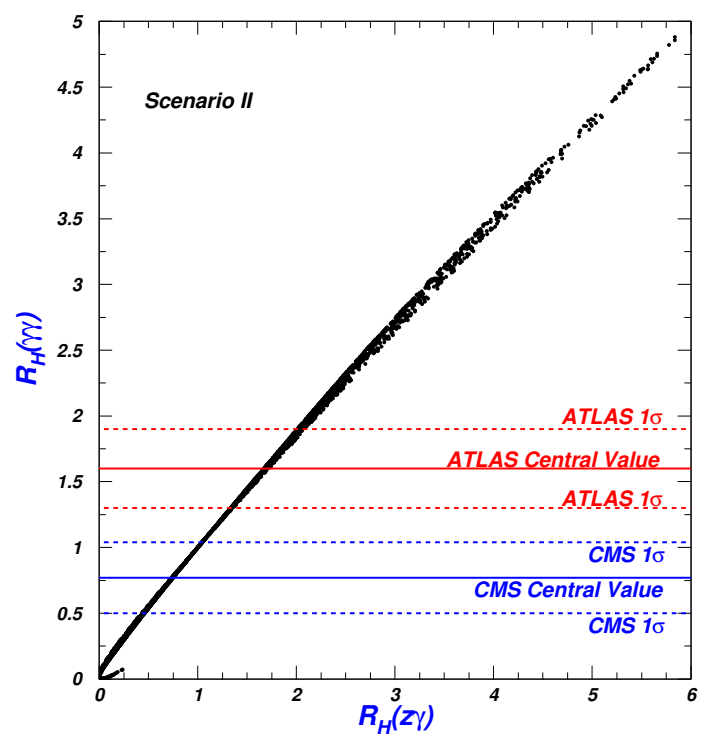

Figure 6. Same as figure 3 , but for $R_{H}(\gamma \gamma)$ versus $R_{H}(Z \gamma)$.

In figure 5 , the samples with $R_{H}(\gamma \gamma)$ being within $1 \sigma$ range of ATLAS and CMS diphoton data are projected on the plane of $a_{2}$ and $m_{H^{ \pm}}$. Figure 5 shows that $-2.7<$ $a_{2}<-0.4$ and $-0.1<a_{2}<1.9$ are respectively favored by the $1 \sigma$ ATLAS and CMS data. The left panel shows that, for $R_{H}(\gamma \gamma)$ is within $1 \sigma$ range of ATLAS diphoton data, the samples lie in the region of $s_{0}^{2}>0.95$, and the vast majority of them congregate the region of $s_{0}^{2}>0.98$. From the right panel, the value of $s_{0}^{2}$ is larger than 0.98 for $R_{H}(\gamma \gamma)$ is within $1 \sigma$ range of CMS diphoton data. The small $m_{H^{ \pm}}$favors a large $s_{0}^{2}$. Due to $R_{H}\left(Z Z^{*}\right) \simeq s_{0}^{2}$ (see eq. (3.3)), the inclusive $Z Z^{*}$ rate is outside $1 \sigma$ range of ATLAS data, but within $1 \sigma$ range of CMS data. Besides, for such large $s_{0}^{2}$, the corresponding $c_{0}^{2}$ is smaller than 0.05 , and the cross section of $e^{+} e^{-} \rightarrow Z h$ is below the upper limit presented by the LEP [72, 73].

In figure 6 , we plot $R_{H}(\gamma \gamma)$ versus $R_{H}(Z \gamma)$. Similar to scenario I, the two rates are also positively correlated. Especially for the region favored by the $1 \sigma$ range of ATLAS and CMS data, the prediction of $R_{H}(Z \gamma)$ equals to that of $R_{H}(\gamma \gamma)$ approximately.

\subsection{Scenario III}

For the scenario III, the observed signal is from the almost degenerate $h$ and $H$. We assume that the mass splitting of $h$ and $H$ is small enough not to be resolve at current statistics, but large enough so that there is hardly interference between the amplitudes of $h$ and $H$, $\left|m_{H}-m_{h}\right| \gg \Gamma(h), \Gamma(H)$ [74]. Therefore, according to eq. (2.10), both the absolute values of $A-C$ and $B$ must be very small, but not to equal to zero exactly. For this case, we can obtain a relation of $m_{H^{ \pm}} \simeq m_{h} \simeq m_{H}$ according to eqs. (2.4), (2.7) and (2.10).

In figure 7 , we plot $R_{h}(\gamma \gamma)+R_{H}(\gamma \gamma)$ versus $a_{2}$ and $c_{0}^{2}$, respectively. We find that the Higgs diphoton rate is suppressed compared to SM value, $0.87<R_{h}(\gamma \gamma)+R_{H}(\gamma \gamma)<0.9$, which is outside $1 \sigma$ range of ATLAS diphoton data, but within $1 \sigma$ range of CMS diphoton data. Due to $a_{1}>0, a_{2}$ must be larger than zero to obtain a very small $|B|(B=$ $\left.-a_{1} v_{d} / 2+a_{2} v_{d} v_{t}\right)$. Thus, $R_{h}(\gamma \gamma)+R_{H}(\gamma \gamma)$ is smaller than 1.0 since the $H^{ \pm}$contributions 

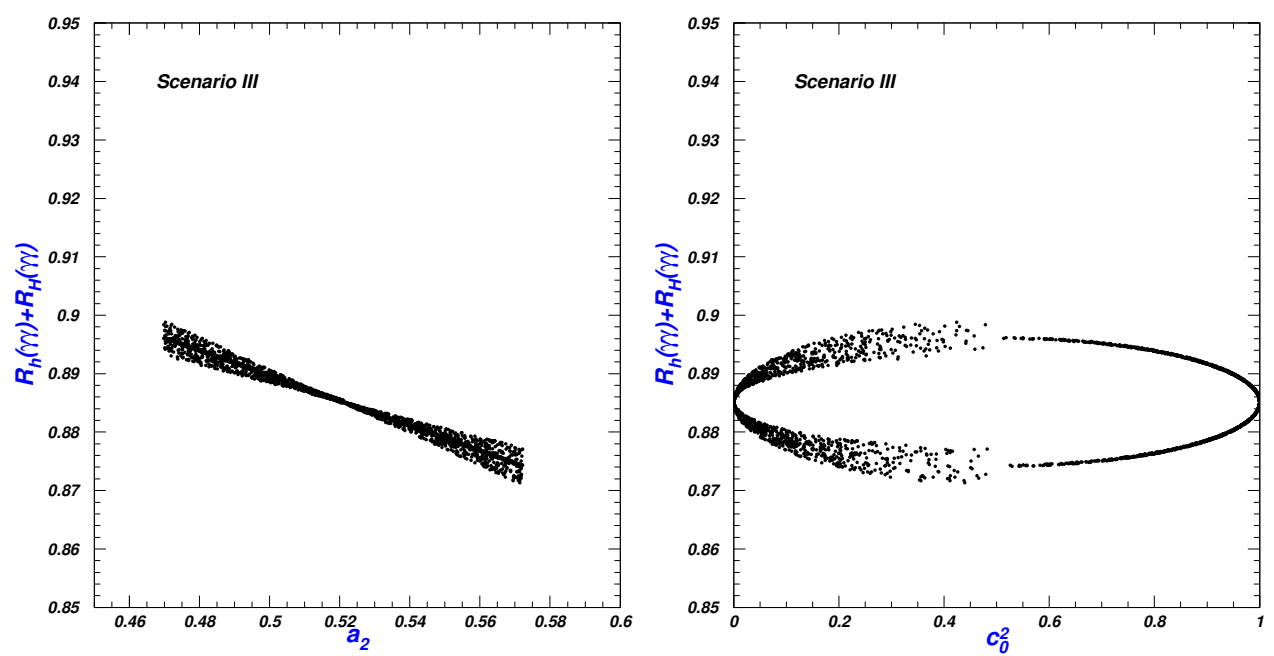

Figure 7. The scatter plots of the parameter space projected on the planes of $R_{h}(\gamma \gamma)+R_{H}(\gamma \gamma)$ versus $a_{2}$ and $c_{0}^{2}$, respectively.

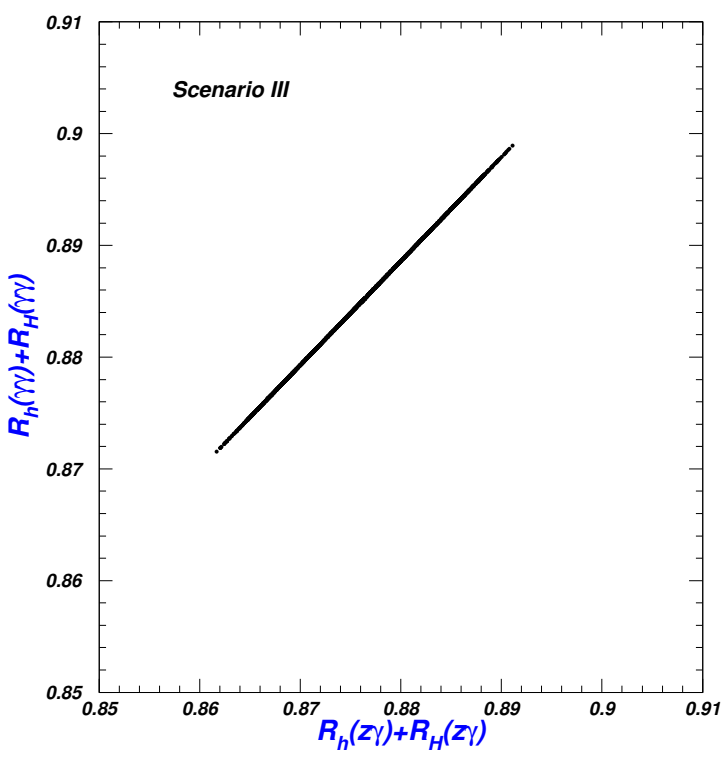

Figure 8. Same as figure 3, but for $R_{h}(\gamma \gamma)+R_{H}(\gamma \gamma)$ versus $R_{h}(Z \gamma)+R_{H}(Z \gamma)$.

will interfere destructively with the $W$ contributions for $a_{2}>0$. The right panel shows that the large mixing angle $\theta_{0}$ may appear. The reason is that $|A-C|$ still may be much smaller than $|B|$ although $|B|$ is very small. Due to $R_{h}\left(Z Z^{*}\right) \simeq c_{0}^{2}$ and $R_{H}\left(Z Z^{*}\right)=s_{0}^{2}$ (see eq. (3.3)), the inclusive $Z Z^{*}$ rate equals to SM prediction value approximately.

In figure 8 , we plot $R_{h}(\gamma \gamma)+R_{H}(\gamma \gamma)$ versus $R_{h}(Z \gamma)+R_{H}(Z \gamma)$. We find that the two rates are also positively correlated, and the correlation is more strong than that of scenario II. $R_{h}(Z \gamma)+R_{H}(Z \gamma)$ is allowed to vary in the narrow region $0.86<R_{h}(Z \gamma)+R_{H}(Z \gamma)<0.89$. 


\section{Conclusion}

In the Higgs triplet model with $\mathrm{Y}=0$, we study the Higgs boson $\gamma \gamma$ and $Z \gamma$ rates at the LHC. We studied three different scenarios: (i) the observed boson is the light Higgs boson $h$; (ii) it is the heavy Higgs boson $H$; (iii) the observed signal is from the almost degenerate $h$ and $H$. We found that, for the first two scenarios, the inclusive Higgs diphoton rates can be enhanced or suppressed compared to the SM value, which is respectively within $1 \sigma$ range of ATLAS and CMS data. For the scenario I, the ATLAS data favors $-3.6<a_{2}<-1.8$ and $m_{H^{ \pm}}<190 \mathrm{GeV}$. The CMS data favors $a_{2}>0$ and allow $a_{2}$ to be smaller than 0 for enough large $m_{H^{ \pm}}$. For the scenario II, the ATLAS and CMS diphoton data favor $-2.7<a_{2}<-0.4$ and $-0.1<a_{2}<1.9$, respectively. For the first two scenarios, the inclusive $Z Z^{*}$ rates are suppressed, which are outside $1 \sigma$ range of ATLAS data and within $1 \sigma$ range of CMS data. For the third scenario, the Higgs diphoton rate is suppressed, which is outside $1 \sigma$ range of ATLAS data, and the $Z Z^{*}$ rate equals to SM value approximately. Besides, the two rates of $h \rightarrow \gamma \gamma$ and $h \rightarrow Z \gamma$ are positively correlated, and they are approximately equal within the $1 \sigma$ range of ATLAS and CMS diphoton data.

\section{Acknowledgments}

This work was supported by the National Natural Science Foundation of China (NNSFC) under grant Nos. 11105116, 11005089, and 11175151.

\section{A The expressions for $\Gamma(h \rightarrow \gamma \gamma)$ and $\Gamma(h \rightarrow Z \gamma)$}

The charged fermion $(f)$, gauge boson $(W)$ and scalar $(s)$ can contribute to the decay widths of $h \rightarrow \gamma \gamma$ and $h \rightarrow Z \gamma$, which are given by [65, 66, 75]

$$
\begin{array}{r}
\Gamma(h \rightarrow \gamma \gamma)=\frac{\alpha^{2} m_{h}^{3}}{256 \pi^{3} v^{2}}\left|\sum_{f} N_{f}^{c} Q_{f}^{2} y_{f} A_{1 / 2}^{\gamma \gamma}\left(\tau_{f}\right)+y_{W} A_{1}^{\gamma \gamma}\left(\tau_{W}\right)+Q_{s}^{2} \frac{v \mu_{h s s^{*}}}{2 m_{s}^{2}} A_{0}^{\gamma \gamma}\left(\tau_{s}\right)\right|^{2} \\
\Gamma(h \rightarrow Z \gamma)=\frac{\alpha^{2} m_{h}^{3}}{128 \pi^{3} s_{W}^{2} c_{W}^{2} v^{2}}\left(1-m_{Z}^{2} / m_{h}^{2}\right)^{3} \mid N_{f}^{c} Q_{f} y_{f} \frac{\left(Q_{R}^{Z}+Q_{L}^{Z}\right)}{2} A_{1 / 2}^{Z \gamma}\left(\tau_{f}, \lambda_{f}\right) \\
+Q_{W} Q_{W}^{Z} y_{W} A_{1}^{Z \gamma}\left(\tau_{W}, \lambda_{W}\right)+\left.Q_{s} Q_{s}^{Z} \frac{v g_{h s s}}{2 m_{s}^{2}} A_{0}^{Z \gamma}\left(\tau_{s}, \lambda_{s}\right)\right|^{2}
\end{array}
$$

where $\tau_{i}=m_{h}^{2} / 4 m_{i}^{2}, \lambda_{i}=m_{Z}^{2} / 4 m_{i}^{2}, Q_{W}=1, Q_{W}^{Z}=c_{W}^{2} . Q_{f, s}$ are the electric charges of fermion and scalar. $N_{f}^{c}$ is the color factor for fermion $f . Q_{R, L(s)}^{Z}=I_{R, L(s)}^{3}-Q_{f(s)} s_{W}^{2}$ with $I_{R, L(s)}^{3}$ being the third isospin components of chiral fermions (scalar). $y_{f}$ and $y_{W}$ denote the Higgs couplings to $f \bar{f}$ and $W W$ normalized to the corresponding SM values. $g_{h s s}$ is the coupling constant of $h s s$. The loop functions $A_{(0,1 / 2,1)}^{\gamma \gamma}$ and $A_{(0,1 / 2,1)}^{Z \gamma}$ in eqs. (A.1) 
and (A.2) are defined as

$$
\begin{aligned}
A_{0}^{\gamma \gamma}(\tau) & =-[\tau-f(\tau)] \tau^{-2}, A_{1 / 2}^{\gamma \gamma}(\tau)=2[\tau+(\tau-1) f(\tau)] \tau^{-2}, \\
A_{1}^{\gamma \gamma}(\tau) & =-\left[2 \tau^{2}+3 \tau+3(2 \tau-1) f(\tau)\right] \tau^{-2}, \\
A_{0}^{Z \gamma}(\tau, \lambda) & =I_{1}(\tau, \lambda), A_{1 / 2}^{Z \gamma}(\tau, \lambda)=-2\left[I_{1}(\tau, \lambda)-I_{2}(\tau, \lambda)\right], \\
A_{1}^{Z \gamma}(\tau, \lambda) & =[2(1+2 \tau)(1-\lambda)+(1-2 \tau)] I_{1}(\tau, \lambda)-8(1-\lambda) I_{2}(\tau, \lambda),
\end{aligned}
$$

where

$$
\begin{aligned}
& I_{1}(\tau, \lambda)=-\frac{1}{(\tau-\lambda)}+\frac{1}{(\tau-\lambda)^{2}}[f(\tau)-f(\lambda)]+\frac{2 \lambda}{(\tau-\lambda)^{2}}[g(\tau)-g(\lambda)], \\
& I_{2}(\tau, \lambda)=\frac{1}{(\tau-\lambda)}[f(\tau)-f(\lambda)]
\end{aligned}
$$

with the functions $f(\tau)$ and $g(\tau)$ given by

$$
\begin{aligned}
& f(\tau)= \begin{cases}\left(\sin ^{-1} \sqrt{\tau}\right)^{2}, & \tau \leq 1 \\
-\frac{1}{4}\left[\log \frac{1+\sqrt{1-\tau^{-1}}}{1-\sqrt{1-\tau^{-1}}}-i \pi\right]^{2}, & \tau>1\end{cases} \\
& g(\tau)= \begin{cases}\sqrt{\tau^{-1}-1}\left(\sin ^{-1} \sqrt{\tau}\right), & \tau \leq 1 \\
\frac{\sqrt{1-\tau^{-1}}}{2}\left[\log \frac{1+\sqrt{1-\tau^{-1}}}{1-\sqrt{1-\tau^{-1}}}-i \pi\right], & \tau>1 .\end{cases}
\end{aligned}
$$

\section{B The vacuum expectation values}

The minimization conditions for the tree-level Higgs potential are

$$
\begin{aligned}
& \left(-\mu^{2}+\lambda_{0} v_{d}^{2}-\frac{a_{1} v_{t}}{2}+\frac{a_{2} v_{t}^{2}}{2}\right) v_{d}=0, \\
& -M_{\Sigma}^{2} v_{t}+b_{4} v_{t}^{3}-\frac{a_{1} v_{d}^{2}}{4}+\frac{a_{2} v_{d}^{2} v_{t}}{2}=0 .
\end{aligned}
$$

Solving the eqs. (B.1) and (B.2) with Mathematica, we can obtain the expressions of $v_{t}$ and $v_{d}$ in terms of the Lagrangian parameters. However, their expressions are very complicated and lengthy. Therefore, we assume $v_{t}$ to be much smaller than 1 , and give the approximate solutions for $a_{2} \mu^{2} \geq 2 M_{\Sigma}^{2} \lambda_{0}$,

$$
\begin{aligned}
& v_{t}=\frac{1}{a_{1}}\left(-\mu^{2}+\frac{a_{1}^{2}}{4 a_{2}}+\frac{2 M_{\Sigma}^{2} \lambda_{0}}{a_{2}}+\frac{\sqrt{-128 \mu^{2} a_{2} M_{\Sigma}^{2} \lambda_{0}+\left(a_{1}^{2}+4 \mu^{2} a_{2}+8 M_{\Sigma}^{2} \lambda_{0}\right)^{2}}}{4 a_{2}}\right) \\
& v_{d}=\sqrt{\frac{M_{\Sigma}^{2}}{a_{2}}+\frac{\mu^{2}}{2 \lambda_{0}}+\frac{a_{1}^{2}}{8 a_{2} \lambda_{0}}+\frac{\sqrt{-128 \mu^{2} a_{2} M_{\Sigma}^{2} \lambda_{0}+\left(a_{1}^{2}+4 \mu^{2} a_{2}+8 M_{\Sigma}^{2} \lambda_{0}\right)^{2}}}{8 a_{2} \lambda_{0}}},
\end{aligned}
$$


and for $a_{2} \mu^{2} \leq 2 M_{\Sigma}^{2} \lambda_{0}$

$$
\begin{aligned}
v_{t} & =\frac{1}{a_{1}}\left(-\mu^{2}+\frac{a_{1}^{2}}{4 a_{2}}+\frac{2 M_{\Sigma}^{2} \lambda_{0}}{a_{2}}-\frac{\sqrt{-128 \mu^{2} a_{2} M_{\Sigma}^{2} \lambda_{0}+\left(a_{1}^{2}+4 \mu^{2} a_{2}+8 M_{\Sigma}^{2} \lambda_{0}\right)^{2}}}{4 a_{2}}\right) \\
v_{d} & =\sqrt{\frac{M_{\Sigma}^{2}}{a_{2}}+\frac{\mu^{2}}{2 \lambda_{0}}+\frac{a_{1}^{2}}{8 a_{2} \lambda_{0}}-\frac{\sqrt{-128 \mu^{2} a_{2} M_{\Sigma}^{2} \lambda_{0}+\left(a_{1}^{2}+4 \mu^{2} a_{2}+8 M_{\Sigma}^{2} \lambda_{0}\right)^{2}}}{8 a_{2} \lambda_{0}}} .
\end{aligned}
$$

From eqs. (B.3) and (B.5), $v_{t}$ approaches to 0 for $a_{1} \rightarrow 0$, which is understandable since $a_{1}$ is the coefficient of the only term in the Lagrangian breaking the custodial symmetry.

Open Access. This article is distributed under the terms of the Creative Commons Attribution License (CC-BY 4.0), which permits any use, distribution and reproduction in any medium, provided the original author(s) and source are credited.

\section{References}

[1] CMS collaboration, Observation of a new boson at a mass of $125 \mathrm{GeV}$ with the CMS experiment at the LHC, Phys. Lett. B 716 (2012) 30 [arXiv:1207.7235] [INSPIRE].

[2] ATLAS collaboration, Observation of a new particle in the search for the standard model Higgs boson with the ATLAS detector at the LHC, Phys. Lett. B 716 (2012) 1 [arXiv: 1207.7214] [INSPIRE].

[3] CDF, D0 collaboration, T. Aaltonen et al., Higgs boson studies at the Tevatron, Phys. Rev. D 88 (2013) 052014 [arXiv:1303.6346] [INSPIRE].

[4] CMS collaboration, Combination of standard model Higgs boson searches and measurements of the properties of the new boson with a mass near $125 \mathrm{GeV}$, CMS-PAS-HIG-13-005 (2013).

[5] ATLAS collaboration, Combined coupling measurements of the Higgs-like boson with the ATLAS detector using up to $25 \mathrm{fb}^{-1}$ of proton-proton collision data, ATLAS-CONF-2013-034 (2013).

[6] CMS collaboration, Search for a light Higgs boson in the $Z$ boson plus a photon decay channel, CMS-PAS-HIG-12-049 (2012).

[7] U. Ellwanger and C. Hugonie, Higgs bosons near $125 \mathrm{GeV}$ in the NMSSM with constraints at the GUT scale, Adv. High Energy Phys. 2012 (2012) 625389 [arXiv:1203.5048].

[8] K. Hagiwara, J.S. Lee and J. Nakamura, Properties of $125 \mathrm{GeV}$ Higgs boson in non-decoupling MSSM scenarios, JHEP 10 (2012) 002 [arXiv:1207.0802] [INSPIRE].

[9] N.D. Christensen, T. Han and S. Su, MSSM Higgs bosons at the LHC, Phys. Rev. D 85 (2012) 115018 [arXiv:1203.3207] [INSPIRE].

[10] B. Kyae and J.-C. Park, A singlet-extension of the MSSM for $125 \mathrm{GeV}$ Higgs with the least tuning, Phys. Rev. D 87 (2013) 075021 [arXiv:1207.3126] [INSPIRE].

[11] J. Cao, Z. Heng, J.M. Yang and J. Zhu, Status of low energy SUSY models confronted with the LHC $125 \mathrm{GeV}$ Higgs data, JHEP 10 (2012) 079 [arXiv:1207.3698] [INSPIRE].

[12] J.-J. Cao, Z.-X. Heng, J.M. Yang, Y.-M. Zhang and J.-Y. Zhu, A SM-like Higgs near $125 \mathrm{GeV}$ in low energy SUSY: a comparative study for MSSM and NMSSM, JHEP 03 (2012) 086 [arXiv:1202.5821] [INSPIRE]. 
[13] J. Cao, Z. Heng, D. Li and J.M. Yang, Current experimental constraints on the lightest Higgs boson mass in the constrained MSSM, Phys. Lett. B 710 (2012) 665 [arXiv:1112.4391] [INSPIRE].

[14] H. An, T. Liu and L.-T. Wang, 125 GeV Higgs boson, enhanced di-photon rate and gauged $U(1)_{P Q}$-extended MSSM, Phys. Rev. D 86 (2012) 075030 [arXiv:1207.2473] [INSPIRE].

[15] M. Berg, I. Buchberger, D. Ghilencea and C. Petersson, Higgs diphoton rate enhancement from supersymmetric physics beyond the MSSM, Phys. Rev. D 88 (2013) 025017 [arXiv: 1212.5009] [INSPIRE].

[16] K. Cheung, C.-T. Lu and T.-C. Yuan, Diphoton rate of the standard-model-like Higgs boson in the extra $\mathrm{U}(1)$ extended MSSM, Phys. Rev. D 87 (2013) 075001 [arXiv:1212.1288] [INSPIRE].

[17] T. Liu, L. Wang and J.M. Yang, Higgs decay to goldstini and its observability at the LHC, Phys. Lett. B 726 (2013) 228 [arXiv:1301.5479] [INSPIRE].

[18] M. Carena, S. Gori, I. Low, N.R. Shah and C.E. Wagner, Vacuum stability and higgs diphoton decays in the MSSM, JHEP 02 (2013) 114 [arXiv:1211.6136] [INSPIRE].

[19] E.J. Chun and P. Sharma, A light triplet boson and Higgs-to-diphoton in supersymmetric type-II seesaw, Phys. Lett. B 722 (2013) 86 [arXiv:1301.1437] [INSPIRE].

[20] T. Kitahara and T. Yoshinaga, Stau with large mass difference and enhancement of the Higgs to diphoton decay rate in the MSSM, JHEP 05 (2013) 035 [arXiv:1303.0461] [INSPIRE].

[21] Z. Kang, Y. Liu and G.-Z. Ning, Highlights of supersymmetric hypercharge \pm 1 triplets, JHEP 09 (2013) 091 [arXiv: 1301.2204] [inSPIRE].

[22] J. Ke, M.-X. Luo, L.-Y. Shan, K. Wang and L. Wang, Searching SUSY leptonic partner at the CERN LHC, Phys. Lett. B 718 (2013) 1334 [arXiv:1207.0990] [INSPIRE].

[23] T. Li, J.A. Maxin, D.V. Nanopoulos and J.W. Walker, A $125.5 \mathrm{GeV}$ Higgs boson in F-SU(5): imminently observable proton decay, a $130 \mathrm{GeV} \gamma$-ray line and SUSY multijets $\& 3$ light stops at the LHC8, Eur. Phys. J. C 72 (2012) 2246 [arXiv:1208.1999] [INSPIRE].

[24] W.-Z. Feng and P. Nath, Higgs diphoton rate and mass enhancement with vectorlike leptons and the scale of supersymmetry, Phys. Rev. D 87 (2013) 075018 [arXiv:1303.0289] [INSPIRE].

[25] A. Delgado, G. Nardini and M. Quirós, A light supersymmetric Higgs sector hidden by a standard model-like Higgs, JHEP 07 (2013) 054 [arXiv: 1303.0800] [INSPIRE].

[26] K. Schmidt-Hoberg, F. Staub and M.W. Winkler, Enhanced diphoton rates at Fermi and the LHC, JHEP 01 (2013) 124 [arXiv: 1211.2835] [INSPIRE].

[27] K. Benakli, M.D. Goodsell and F. Staub, Dirac gauginos and the $125 \mathrm{GeV}$ Higgs, JHEP 06 (2013) 073 [arXiv: 1211.0552] [INSPIRE].

[28] L. Wang and J.M. Yang, The LHC di-photon Higgs signal predicted by little Higgs models, Phys. Rev. D 84 (2011) 075024 [arXiv:1106.3916] [INSPIRE].

[29] L. Wang and J.M. Yang, Higgs boson decays and production via gluon fusion at LHC in littlest Higgs models with T-parity, Phys. Rev. D 79 (2009) 055013 [arXiv:0812.4609] [INSPIRE].

[30] T. Han, H.E. Logan, B. McElrath and L.-T. Wang, Loop induced decays of the little Higgs: $H \rightarrow g g, \gamma \gamma$, Phys. Lett. B 563 (2003) 191 [Erratum ibid. B 603 (2004) 257-259] [hep-ph/0302188] [INSPIRE]. 
[31] C.-R. Chen, K. Tobe and C.-P. Yuan, Higgs boson production and decay in little Higgs models with T-parity, Phys. Lett. B 640 (2006) 263 [hep-ph/0602211] [INSPIRE].

[32] J. Reuter and M. Tonini, Can the 125 GeV Higgs be the little Higgs?, JHEP 02 (2013) 077 [arXiv: 1212.5930] [INSPIRE].

[33] X.-F. Han, L. Wang, J.M. Yang and J. Zhu, Little Higgs theory confronted with the LHC Higgs data, Phys. Rev. D 87 (2013) 055004 [arXiv:1301.0090] [INSPIRE].

[34] L. Wang, J. M. Yang and J. Zhu, Dark matter in little Higgs model under current experimental constraints from LHC, Planck and Xenon, Phys. Rev. D 88 (2013) 075018 [arXiv: 1307.7780] [INSPIRE].

[35] G. Burdman, C.E. Haluch and R.D. Matheus, Is the LHC observing the pseudo-scalar state of a two-Higgs doublet model?, Phys. Rev. D 85 (2012) 095016 [arXiv:1112.3961] [InSPIRE].

[36] X.-G. He, B. Ren and J. Tandean, Hints of standard model Higgs boson at the LHC and light dark matter searches, Phys. Rev. D 85 (2012) 093019 [arXiv: 1112.6364] [INSPIRE].

[37] A. Arhrib, R. Benbrik and C.-H. Chen, $H \rightarrow \gamma \gamma$ in the complex two Higgs doublet model, arXiv: 1205.5536 [INSPIRE].

[38] L. Wang and X.-F. Han, LHC diphoton Higgs signal and top quark forward-backward asymmetry in quasi-inert Higgs doublet model, JHEP 05 (2012) 088 [arXiv:1203.4477] [INSPIRE].

[39] S. Chang et al., Comprehensive study of two Higgs doublet model in light of the new boson with mass around $125 \mathrm{GeV}, \mathrm{JHEP} 05$ (2013) 075 [arXiv:1210.3439] [INSPIRE].

[40] N. Chen and H.-J. He, LHC signatures of two-Higgs-doublets with fourth family, JHEP 04 (2012) 062 [arXiv:1202.3072] [INSPIRE].

[41] T. Abe, N. Chen and H.-J. He, LHC Higgs signatures from extended electroweak gauge symmetry, JHEP 01 (2013) 082 [arXiv:1207.4103] [INSPIRE].

[42] C. Han, N. Liu, L. Wu, J.M. Yang and Y. Zhang, Two-Higgs-doublet model with a color-triplet scalar: a joint explanation for top quark forward-backward asymmetry and Higgs decay to diphoton, Eur. Phys. J. C 73 (2013) 2664 [arXiv:1212.6728] [INSPIRE].

[43] C.-W. Chiang and K. Yagyu, Implications of Higgs boson search data on the two-Higgs doublet models with a softly broken $Z_{2}$ symmetry, JHEP 07 (2013) 160 [arXiv:1303.0168] [INSPIRE].

[44] R. Jora, S. Nasri and J. Schechter, Naturalness in a simple two Higgs doublet model, Int. J. Mod. Phys. A 28 (2013) 1350036 [arXiv:1302.6344] [InSPIRE].

[45] L. Wang and X.-F. Han, Status of the aligned two-Higgs-doublet model confronted with the Higgs data, arXiv:1312.4759 [INSPIRE].

[46] A. Celis, V. Ilisie and A. Pich, LHC constraints on two-Higgs doublet models, JHEP 07 (2013) 053 [arXiv: 1302.4022] [INSPIRE].

[47] W.-F. Chang, J.N. Ng and J.M. Wu, Constraints on new scalars from the LHC $125 \mathrm{GeV}$ Higgs signal, Phys. Rev. D 86 (2012) 033003 [arXiv:1206.5047] [INSPIRE].

[48] M. Chala, $h \rightarrow \gamma \gamma$ excess and dark matter from composite Higgs models, JHEP 01 (2013) 122 [arXiv:1210.6208] [INSPIRE].

[49] A. Akeroyd and S. Moretti, Enhancement of $H \rightarrow \gamma \gamma$ from doubly charged scalars in the Higgs triplet model, Phys. Rev. D 86 (2012) 035015 [arXiv:1206.0535] [inSPIRE].

[50] A. Arhrib, R. Benbrik, M. Chabab, G. Moultaka and L. Rahili, Higgs boson decay into 2 photons in the type II seesaw model, JHEP 04 (2012) 136 [arXiv:1112.5453] [INSPIRE]. 
[51] L. Wang and X.-F. Han, The recent Higgs boson data and Higgs triplet model with vector-like quark, Phys. Rev. D 86 (2012) 095007 [arXiv:1206.1673] [INSPIRE].

[52] L. Wang and X.-F. Han, $130 \mathrm{GeV} \gamma$-ray line and enhancement of $h \rightarrow \gamma \gamma$ in the Higgs triplet model plus a scalar dark matter, Phys. Rev. D 87 (2013) 015015 [arXiv:1209.0376] [INSPIRE].

[53] Y. Kajiyama, H. Okada and K. Yagyu, Two loop radiative seesaw model with inert triplet scalar field, Nucl. Phys. B 874 (2013) 198 [arXiv:1303.3463] [INSPIRE].

[54] P. Bhupal Dev, D.K. Ghosh, N. Okada and I. Saha, $125 \mathrm{GeV}$ Higgs boson and the type-II seesaw model, JHEP 03 (2013) 150 [Erratum ibid. 1305 (2013) 049] [arXiv:1301.3453] [INSPIRE].

[55] F. Arbabifar, S. Bahrami and M. Frank, Neutral Higgs bosons in the Higgs triplet model with nontrivial mixing, Phys. Rev. D 87 (2013) 015020 [arXiv:1211.6797] [INSPIRE].

[56] C. Englert, E. Re and M. Spannowsky, Triplet Higgs boson collider phenomenology after the LHC, Phys. Rev. D 87 (2013) 095014 [arXiv: 1302.6505] [INSPIRE].

[57] A. Melfo, M. Nemevsek, F. Nesti, G. Senjanovic and Y. Zhang, Type II seesaw at LHC: the roadmap, Phys. Rev. D 85 (2012) 055018 [arXiv:1108.4416] [INSPIRE].

[58] Y. Cai, W. Chao and S. Yang, Scalar septuplet dark matter and enhanced $h \rightarrow \gamma \gamma$ decay rate, JHEP 12 (2012) 043 [arXiv:1208.3949] [INSPIRE].

[59] J. Cao, P. Wan, J.M. Yang and J. Zhu, The SM extension with color-octet scalars: diphoton enhancement and global fit of LHC Higgs data, JHEP 08 (2013) 009 [arXiv:1303.2426] [INSPIRE].

[60] D.A. Ross and M.J.G. Veltman, Neutral currents in neutrino experiments, Nucl. Phys. B 95 (1975) 135 [inSPIRE].

[61] J.F. Gunion, R. Vega and J. Wudka, Higgs triplets in the standard model, Phys. Rev. D 42 (1990) 1673 [INSPIRE].

[62] C.-W. Chiang and K. Yagyu, Higgs boson decays to $\gamma \gamma$ and $Z \gamma$ in models with Higgs extensions, Phys. Rev. D 87 (2013) 033003 [arXiv:1207.1065] [INSPIRE].

[63] B. Coleppa, K. Kumar and H.E. Logan, Can the 126 GeV boson be a pseudoscalar?, Phys. Rev. D 86 (2012) 075022 [arXiv: 1208.2692] [INSPIRE].

[64] J. Cao, L. Wu, P. Wu and J.M. Yang, The $Z+$ photon and diphoton decays of the Higgs boson as a joint probe of low energy SUSY models, JHEP 09 (2013) 043 [arXiv:1301.4641] [INSPIRE].

[65] C.-S. Chen, C.-Q. Geng, D. Huang and L.-H. Tsai, $h \rightarrow Z \gamma$ in type-II seesaw neutrino model, Phys. Lett. B 723 (2013) 156 [arXiv: 1302.0502] [InSPIRE].

[66] C.-S. Chen, C.-Q. Geng, D. Huang and L.-H. Tsai, New scalar contributions to $h \rightarrow Z \gamma$, Phys. Rev. D 87 (2013) 075019 [arXiv: 1301.4694] [INSPIRE].

[67] P. Fileviez Perez, H.H. Patel, M. Ramsey-Musolf and K. Wang, Triplet scalars and dark matter at the LHC, Phys. Rev. D 79 (2009) 055024 [arXiv:0811.3957] [INSPIRE].

[68] S. Kanemura and K. Yagyu, Radiative corrections to electroweak parameters in the Higgs triplet model and implication with the recent Higgs boson searches, Phys. Rev. D 85 (2012) 115009 [arXiv:1201.6287] [InSPIRE].

[69] ALEPH collaboration, A. Heister et al., Search for charged Higgs bosons in $e^{+} e^{-}$collisions at energies up to $\sqrt{s}=209 \mathrm{GeV}$, Phys. Lett. B 543 (2002) 1 [hep-ex/0207054] [INSPIRE]. 
[70] DELPHI collaboration, J. Abdallah et al., Search for charged Higgs bosons at LEP in general two Higgs doublet models, Eur. Phys. J. C 34 (2004) 399 [hep-ex/0404012] [INSPIRE].

[71] A. Djouadj, J. Kalinowski and M. Spira, HDECAY: a program for Higgs boson decays in the standard model and its supersymmetric extension, Comput. Phys. Commun. 108 (1998) 56 [hep-ph/9704448] [INSPIRE].

[72] LEP Working Group for Higgs boson searches, ALEPH, DELPHI, L3, OPAL collaboration, R. Barate et al., Search for the standard model Higgs boson at LEP, Phys. Lett. B 565 (2003) 61 [hep-ex/0306033] [INSPIRE].

[73] Aleph, Delphi, L3, OPAL, LeP Working Group for Higgs Boson Searches collaboration, S. Schael et al., Search for neutral MSSM Higgs bosons at LEP, Eur. Phys. J. C 47 (2006) 547 [hep-ex/0602042] [INSPIRE].

[74] B. Batell, D. McKeen and M. Pospelov, Singlet neighbors of the Higgs boson, JHEP 10 (2012) 104 [arXiv:1207.6252] [INSPIRE].

[75] A. Djouadi, The Anatomy of electro-weak symmetry breaking. II. The Higgs bosons in the minimal supersymmetric model, Phys. Rept. 459 (2008) 1 [hep-ph/0503173] [INSPIRE]. 